\title{
Potential sources of error when applying a corneal reflex eye-monitoring technique to infant subjects
}

\author{
GORDON W. BRONSON \\ Mills College, Oakland, California 94613
}

\begin{abstract}
Because infant head movements cannot be severely restrained, and because calibration procedures are difficult to conduct, infant scanning data are subject to a number of sources of latent error. Here, sources of potential error are identified, and suggestions are offered for minimizing or eliminating their effects.
\end{abstract}

A number of laboratories recently have begun to use bright-pupil/corneal reflex techniques for the study of infant scanning patterns (e.g., Aslin, 1981; Bronson, 1982; Hainline, 1981b). The approach promises to clarify a variety of longstanding issues concerning the nature of early visual processing, and one can anticipate an expanding number of infant studies based upon this technique. However, extending to infant subjects a procedure originally developed for use on adults raises a number of difficulties that are nonexistent or trivial when working with older subjects. This article focuses on those aspects of system design, and of calibration procedure, that are of particular concern when applying the corneal reflex technique to infant subjects. The aim is to guide the development of future systems and to aid in detecting potential artifacts in existing data.

Among the various methods that have been developed for the more or less accurate determination of the loci of successive fixations as a subject scans over the visual field, the bright-pupil/corneal reflex technique appears to hold the greatest promise for application to infant subjects and, hence, is the primary focus of the present article (for a general review of the various methods, see Young \& Sheena, 1975). Since the rather detailed analyses to follow are of interest mainly to those who already are using, or are about to apply, this type of technique, it is assumed that the reader has a general familiarity with the method. (Details of its application to infant subjects can be found in Bronson, 1982, and in Hainline, 1981a). Briefly, an infrared light source (largely invisible to the subjects, but not to the recording camera) is aligned with the camera axis. Light reflected from the retina back to the camera makes the pupil appear brighter than the surrounding eye and face, hence the "bright-pupil" designation. The light source also appears as a small bright spot reflected back from the front surface of the eye (the "corneal reflex"). If the optic axis of the eye happens to be aimed directly

Address reprint requests to the author at the Department of Psychology, Mills College, Oakland, California 94613. toward the camera, the reflected spot will appear to be located right in the center of the pupil; in other circumstances, the displacement of the spot from the pupil center will be a function of the degree to which the optic axis of the eye is turned away from the camera location. Thus, measures derived from the displacement of the corneal reflex with respect to the center of the pupil can indicate the momentary direction of gaze. (This geometry is illustrated in Figure 5 below.)

Two general sources of error can arise when applying this technique to infant subjects. First, infant subjects typically object to tight head constraints, yet when even a rather small amount of head motion is permitted, parallax effects, or distorted video images, can introduce undetected errors into the scanning data. The first section of this article will illustrate these various effects and discuss ways in which they can be minimized or eliminated. Second, when using a corneal reflex method, the raw data specifying spot/pupil-center displacements subsequently must be transformed into measures representing the actual loci of successive fixations within the visual field. With an adult subject, the collection of the necessary "calibration data" poses no great difficulty: The subject need only be instructed to look precisely at a selected sequence of specific points within the visual field. From such calibration data, it then is possible to derive an algorithm for determining where the subject is looking during intervals of "free scanning" over the stimulus (see Sheena \& Borah, 1981). When the subject is a noninstructable infant, however, the collection of the necessary calibration data becomes more difficult, and the information gathered often is rather limited and of uncertain quality. The second section of the article discusses issues that arise in transforming raw scanning data into locus-of-fixation measures when working with relatively limited and rather unreliable calibration data.

\section{PARALLAX EFFECTS}

The following sections examine various sources of parallax error that might occur when some degree of 
free head movement is allowed. The most obvious (and easily corrected) effects are considered first, and then more subtle sources of parallax error are examined. The calculations indicating the magnitudes of the errors that might be associated with the various effects will assume optical distances that could be typical for an infant eye-monitoring system; the magnitudes can be considerably different, however, under other spatial arrangements.

\section{Stimulus Distance}

The expositions of parallax effects can be illustrated most clearly by assuming that as the subject's head is translated sidewise or vertically, the eye is being rotated so that the optic axis remains directed toward the camera. In this case, the data indicate no change in the locus of fixation, since the corneal spot remains in the center of the pupil. During such a movement, however, the subject is actually fixating whatever part of the stimulus field happens to be aligned with the camera. ${ }^{1}$ Comparison of Figures $1 \mathrm{a}$ and $\mathrm{lb}$ show that if the stimulus is not at the same optical distance as the camera, the locus of regard within the stimulus will in fact have shifted. One can determine the magnitude of the possible error from geometry of the physical arrangement. For example, if the camera is $50 \mathrm{~cm}$ from the subject and the stimulus at $40 \mathrm{~cm}$, and the head is moved some $4 \mathrm{~cm}$, the error on the stimulus field $\Delta \mathrm{x}$ is $.8 \mathrm{~cm}$, an error of about $1.1 \mathrm{deg}$ of visual angle.

\section{Infrared-Source Distance}

To avoid a second type of parallax effect, the optical distance from the eye to the infrared light source (typically, the condenser lens that focuses the light) and to the camera lens should be equal. When this is the case, as in Figure 2a, if the head is moved while the optic axis of the eye remains directed toward the camera, the reflection of the infrared source will remain in the center of the pupil-indicating, correctly, that the locus of regard has remained unchanged. If the distances are unequal, however, the data will indicate, spuriously, that the optic axis has shifted away from the camera when the head is moved. To analyze this more complex situation only the "head moved" condition need be illustrated, as in Figure 2b. Here, when the optic axis remains directed toward the camera during a head translation, the corneal spot is displaced from the pupil center by an amount $\Delta \mathrm{x}$, indicating, erroneously, that the subject has shifted his fixation. From the geometry of the situation, given a camera distance of $50 \mathrm{~cm}$ and an infrared source $60 \mathrm{~cm}$ from the eye, and assuming a typical infant radius of corneal curvature of $7 \mathrm{~mm}$, a head movement of $4 \mathrm{~cm}$ produces an apparent shift of regard of about $6 \mathrm{deg}$ of visual angle.

\section{Tracking Mirror Effects}

The introduction of a tracking mirror into the system, thus allowing the eye to be kept within the camera
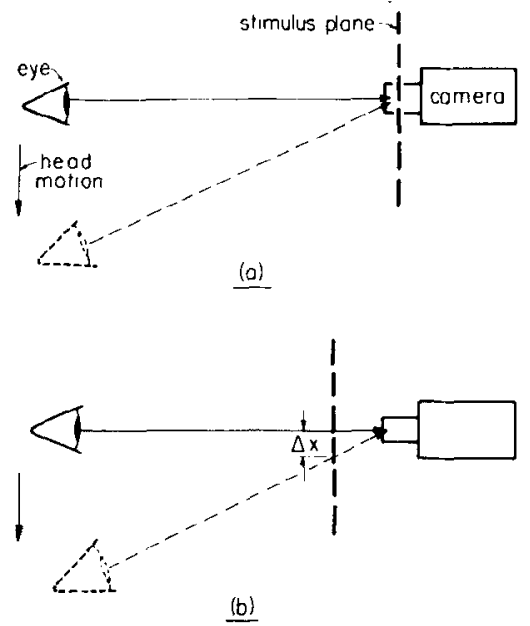

Figure 1. Parallax effects associated with the location of the stimulus plane. In Figure 1a, the camera and stimulus plane are equally distant from the subject; in Figure 1b, the stimulus plane is closer to the subject, resulting in a parallax error $\Delta \mathbf{x}$. (Not shown in the figure is a half-mirror that sometimes is introduced so that the camera and the stimulus are at physically different locations; only the optical distances are relevant for this analysis.)

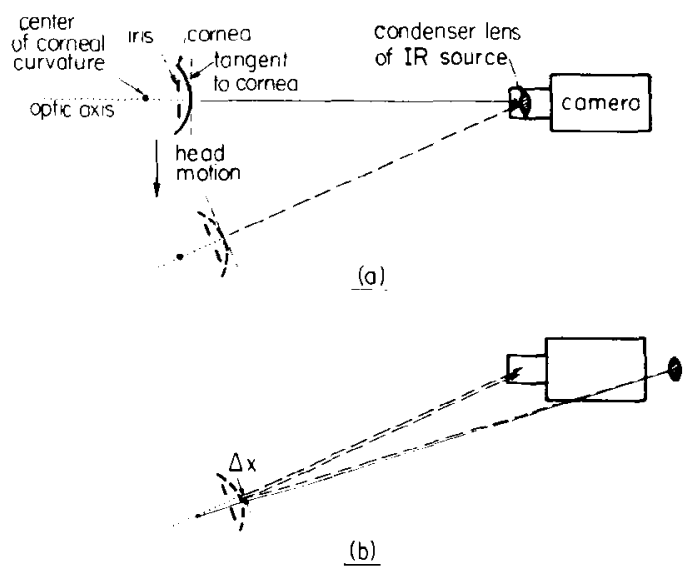

Figure 2. Parallax effects as a function of the location of the infrared (IR) light source. In Figure 2a, the IR source is at the same optical distance as the camera; in Figure 2b, it is located at a greater optical distance. In $2 b$ the corneal reflection is displaced from the optic axis by an amount, $\Delta x$, equal to one-half of the distance to a line connecting the IR source and the center of comeal curvature. (The half-mirror that is used to combine the optical pathways of the camera and the IR source has been omitted from the illustration, since only the relative lengths of the optical pathways need be considered.)

field when the head is translated, raises a number of additional parallax issues. These are considered in order of increasing complexity.

Eye-locating mirror. The most simple case of a mirror used only to correct for differing initial placements of the subjects within the apparatus raises no problems. If the mirror is then left unchanged throughout the 
remainder of the experiment, and each subject is calibrated individually, it can be located at any point between the subject and the camera. Should it be necessary to make additional adjustments within the course of an individual experiment, however, or if the calibration parameters for mapping an infant's eye movements onto the stimulus are not determined individually for each subject, then the considerations outlined in the following sections will apply.

Eye-tracking mirror. As in the earlier analyses, the exposition can be simplified by assuming that as the head is moved the eye is rotated in a manner that keeps the optic axis of the eye directed toward the camera. When the tracking mirror is adjusted to hold the pupil in the center of the camera field, no parallax effects will result if the mirror is located at the same optical distance as the stimulus plane. Thus, as shown in Figure 3a, when the head is shifted the data will indicate, correctly, no change in the locus of fixation on the stimulus. In the circumstance illustrated in Figure $3 b$, however, the data would show no change in fixation when the optic axis remains directed toward the camera, however, the subject would be viewing a different location on the stimulus. For example, if the tracking mirror is at $50 \mathrm{~cm}$ with the stimulus plane $40 \mathrm{~cm}$ from the subject's eye, and if the mirror is rotated to keep the eye in the center of the camera field during a 4-cm head movement, the data

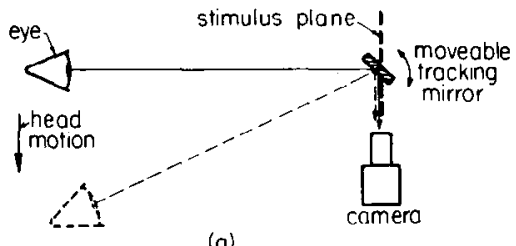

$\underline{(a)}$

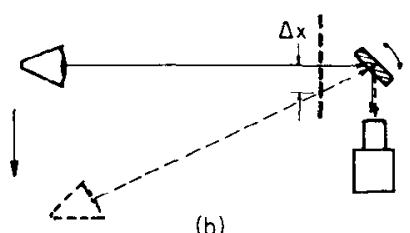

(b)

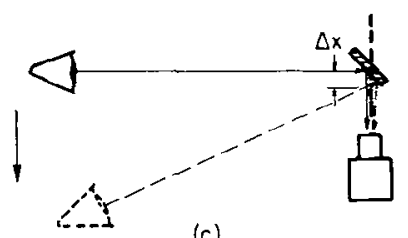

(c)

Figure 3. Parallax effects as a function of the location of a movable tracking mirror. In Figure 3a, the tracking mirror and stimulus plane are equally distant from the subject; in Figure 3b, the stimulus plane is closer; and in Figure 3c, the arrangement is as in $3 \mathrm{a}$ but the mirror remains unmoved as the head is translated. In $3 b$ a parallax error $\Delta x$ results from the differing optical distances. In $3 c$ the error $\Delta x$ is due to a shift of the optical pathway to a different location on the mirror. will indicate no shift of regard, although the location of fixation on the stimulus will in fact have changed by some $1.1 \mathrm{deg}$ of visual angle. (The analysis here is identical to that derived for the Figure 1 conditions, and comparison between Figures 1a and 3 a will show that when a movable mirror is to be introduced into the system the mirror, not the camera, should be located in the plane of the stimulus.)

An additional type of error can occur, even with the ideal mirror location of Figure 3a, when the tracking mirror fails to accurately keep pace with a head movement. The effect can most easily be illustrated by assum. ing that the mirror simply was left unchanged during a small head movement, as shown in Figure 3c. In this circumstance, if the optic axis of the eye is to remain directed toward the camera the optical path from the subject's eye to the camera lens will have to fall on a different location on the mirror, and therefore also on the stimulus plane; the data would show, erroneously, "no change" in the locus of fixation. For example, given a mirror distance of $40 \mathrm{~cm}$ and a camera lens at $60 \mathrm{~cm}$, during a $4-\mathrm{cm}$ head movement the fixation on the mirror, and on the stimulus plane, must have shifted by a $\Delta x$ of $1.3 \mathrm{~cm}$, introducing an error in the apparent locus of fixation of some $2 \mathrm{deg}$ of visual angle. It is evident, therefore, that if a system is to include a tracking mirror that is to be readjusted during the course of an experiment, it should be controlled automatically: To avoid error, it must keep the eye accurately centered within the camera field, and human reaction times will be too slow to closely follow unanticipated head movements. (In addition, a manually controlled mirror is likely to introduce rather serious errors of a second kind, as will be pointed out in a later section.)

Two eye-tracking mirrors. Since continuously adjustable tracking mirrors must compensate for subjects' head movements in both the horizontal and vertical directions, a further difficulty is introduced. The seemingly simple solution of using a single mirror gimballed to track movements in both directions is not fully satisfactory. In one of the two dimensions, a mirror adjustment inevitably will rotate the image that is transmitted to the camera. If the image is rotated, then a saccade in, say, a horizontal direction will appear to have traversed obliquely across the stimulus. To avoid a rotation of the video image, the axis of mirror rotation must be at right angles to the optical pathway and lie in the plane of the mirror. These conditions can be approximated by introducing two separate tracking mirrors, one for each direction of head movement, but only if the mirrors are spaced rather far apart within the optical pathway. As illustrated in Figures 3a and 3b, however, each of the mirrors should be located in the plane of the stimulus, a condition that cannot now be satisfied. To cope with the difficulty that is inherent in the use of two separate tracking mirrors, I have experimented successfully with a liquid prism. Two glass plates are separated by a flex- 
ible bellows, and the unit is filled with a clear liquid of high refractive index. One glass face is pivoted to rotate about a horizontal axis, and the other about a vertical axis, producing, in effect, two variable wedge prisms. The unit is capable of deflecting light in any direction, and, of particular importance here, the effective point of deflection always lies between the two glass plates. ${ }^{2}$ In consequence, the parallax errors illustrated in Figure 3b can essentially be avoided if the midpoint of the prism and the stimulus plane are set at the same optical distance from the subject's eye.

Summary. An on-line tracking of the infant's head movements will reduce the incidence of "off-camera" intervals, providing more complete data sets, and it also might permit the recording of a smaller area of the face, thereby increasing the size of the image and, hence, the precision of measurement. To avoid the introduction of parallax effects as the optical path is deflected, the point of deflection and the plane of the stimulus should be equally distant from the subejct's eye and the control must be such that the eye is kept closely centered within the camera field. These conditions can be met if a variable liquid prism is used for optical deflection, and the system is automatically controlled to maintain an accurate centering of the eye in the camera field.

\section{VIDEO DISTORTION}

In addition to the possible introduction of parallax effects, permitting some degree of free head movement introduces a second source of potential error when images of the eye are being collected with a vidicon sensor. The electronic scan that develops each video field lasts for about 1/60 sec, and the image of the eye may have moved appreciably during this interval. In consequence, rapid horizontal head movements would result in geometric pupil distortions of the sort illustrated in Figure 4. (Analogous effects will follow from vertical head motions, but only the horizontal case need be discussed.)

When the subject is regarding a part of the stimulus that lies near to the camera axis, no serious error is introduced. For instance, in the case illustrated in Figure 4a both the "actual" and the recorded image would indicate that the subject is looking directly toward the camera. When the subject is looking considerably above or below the camera axis, however, a horizontal head movement will alter differentially the apparent center of the pupil and the apparent spot location. In Figure $4 \mathrm{~b}$, for example, the subject is looking downward during a head motion to the right, and the motioninduced shift in the apparent pupil center is greater than the change induced in the spot location by an amount $\Delta \mathrm{x}$. Analogous results will occur when the subject is looking far above the camera axis.

An analysis of data collected on 2- to 5-month-old infants shows that during intervals of continuous atten-

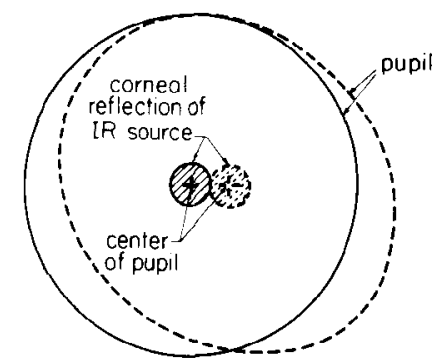

(0)

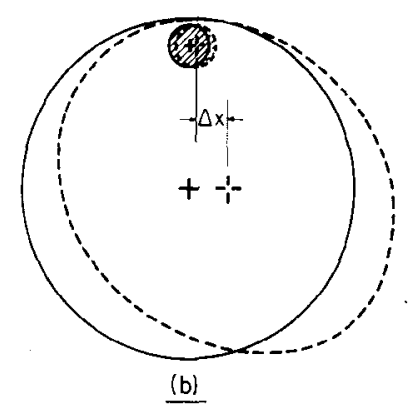

Figure 4. Geometric distortions of the video image due to rapid head movements. Solid lines indicate the actual pupil/ spot configurations; dotted lines show the recorded video images. In Figure $4 a$, the subject is looking directly toward the camera; although the pupil takes an elliptical shape, the spot location shifts in a manner that keeps it in the "center" of the ellipse. In Figure 4b, the subject is looking directly below the camera; the apparent pupil center again is shifted by the head movement, but in this circumstance the spot location is relatively unchanged, resulting in an apparent horizontal displacement of the spot from the vertical axis of the pupil, $\Delta x$.

tion to the stimulus, an infant's free head movement often may reach a peak speed of around $5 \mathrm{~cm} / \mathrm{sec}^{3}$ From the geometry of the situation, one can calculate the magnitude of the consequent error. If the image of the pupil subsumes some 40 of the 256 video lines, for the situation illustrated in Figure $4 \mathrm{~b}$ such movements will produce an error in the measured direction of regard of about $.8 \mathrm{deg}$ of visual angle. Moreover, the effect due to geometric distortion will increase as a linear function of the number of video lines subsumed by the pupil; therefore, should measurement precision be enhanced by recording a larger image of the pupil, there will be a directly corresponding increase in the magnitude of these errors.

Finally, notice that motion of the image over the video field also can be induced by sudden readjustments of an eye-tracking mirror (or variable prism): for example, when attempting to quickly recenter a pupil that has moved to the edge of the camera's field of view. If done manually the induced motion in the camera image might in fact be very rapid, producing a transient error, an apparent brief shift in the locus of fixation, of considerable magnitude. This constitutes a second argument for an automated control system 
should an eye-tracking unit be included in the optical pathway. The data show that infant head movements are ramped; that is, they start slowly, reach a peak velocity, and then slow to a stop. Therefore, an accurate control system not only can avoid a too sudden readjustment of the tracking mirror, but also can actually minimize the speed of motion of the image over the video field during infant-initiated head movements.

\section{CORNEAL CURVATURE EFFECTS}

Raw scanning data indicate only changes in the spot/pupil-center relationship and, subsequently, must be translated into measures indicative of discrete fixations directed to particular locations within the stimulus field. When working with adults, the collection of the calibration data for making such translations is relatively easy: The subject simply is directed to look precisely at a variety of specific points deployed over the visual field. From such arrays of relatively precise data, one can develop fairly accurate algorithms for translating raw scanning data into locus-of-regard measurements (see Sheena \& Borah, 1981). Infants, however, may not always be attentive to the small "calibration" targets, thus limiting the amount of easily collectible data. Furthermore, very young infants appear to have an inherent difficulty in precisely centering fixations on some discrete stimulus feature, making the data unreliable (see Bronson, 1982; Harris, Hainline, \& Abramov, 1981). In brief, the calibration data collected on infant subjects typically has proved to be limited and rather imprecise. This has made it difficult to estimate empirically (i.e., on the basis of calibration data) the magnitudes of several phenomena that affect the translation of raw data into specific stimulus fixations.

Two major kinds of issue arise. First, as is the case with adults, the infant fovea is somewhat displaced from the optic axis. Thus, for example, when the corneal spot appears to be right in the center of the image of the pupil; the infant may actually be fixating a point roughly $6 \mathrm{deg}$ above and $8 \mathrm{deg}$ to one side of the camera location (see Bronson, 1982; Slater \& Findlay, 1972). At present, however, the above values remain only very rough estimates, and the degree of individual variation in foveal displacement is unknown. ${ }^{4}$ This "foveal displacement" issue and the calibration problems it engenders have been discussed in some detail elsewhere (Bronson, 1982) and need not be reexamined here. A second major difficulty in the calibration of infant data remains largely unconsidered, however, and warrants a close analysis. Here, the issue centers on effects associated with variations in the radius of corneal curvature. As the optic axis of the eye is turned toward off-camera locations, the apparent location of the reflected corneal spot will be shifted off the axis, and the magnitude of the shift will depend on several parameters of corneal curvature. When working with adult subjects, the com- plex of effects produced by variations in corneal curvature can be dealt with (indirectly) simply by collecting accurate calibration data on fixations directed to a set of locations that are appropriately spaced over the visual field. Sheena and Borah (1981) have presented a sophisticated analytic technique for the subsequent processing of this kind of adult calibration data. However, because the comparable sets of infant calibration data are likely to be of limited scope and unreliable quality, they usually cannot support this type of fine-grained analysis. This raises a number of questions regarding the optional treatment of infant calibration data: (1) It is appropriate to combine and average the calibration data collected on different subjects; that is, will the reliability of the data thereby be enhanced, or might it be reduced due to large individual variations in corneal curvature? (2) How much error is introduced when calibration data have been collected only from fixation points located near to (or far from) the camera position and then are applied to cases when an infant's fixations are directed to more peripheral (or proximal) areas of the visual field; that is, to what extent might variations in the radius of corneal curvature at different eccentricities from the optic axis introduce a serious nonlinearity into the calibration algorithm? And (3) are calibration data collected from a set of fixations along, say, the horizontal axis of the visual field appropriate for interpreting scanning data on fixations that vary along a vertical dimension; that is, is the radius of corneal curvature usually about the same across different meridians of the infant cornea? This final section will examine these issues analytically, using published data on the shapes of infant corneas. These data come from photokeratoscopic studies conducted on very young babies by Mandell (1967) and York and Mandell (1968).

\section{Individual Variations in Corneal Curvature}

Figure 5 shows that the distance $\mathrm{k}$ is a major variable linking the observed spot/pupil-center displacement, $x$, to the angle of regard, $\theta$. Although variations in radius of curvature may not be related precisely to individual differences in the value of $\mathrm{k}$ (since individual corneairis distances also may vary somewhat; see Figure 5),

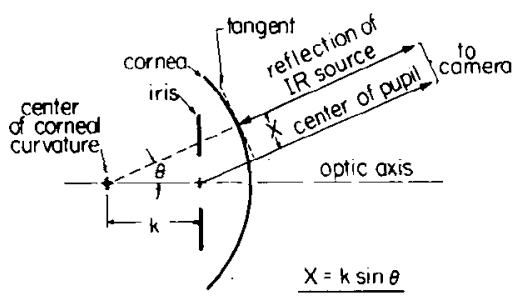

Figure 5. A schematic representation of the determinants of spot/pupil-center relationships. The displacement of the spot from the center of the pupil, $x$, will be a function of $k$ and of the angle $\theta$ between the camera location and the optic axis of the eye. 
curvature differences can be used to make an initial estimate of the general range of infant variability in the $\mathrm{k}$ parameter. The York and Mandell (1968) data report that among infants under 10 months of age, the radius at the center of the cornea ranged from about 6.5 to $7.8 \mathrm{~mm}$ and, generally, seemed unrelated to infant age (beyond the 1st year, however, the range of individual variation had decreased considerably). Assuming that the pupil is located about $2 \mathrm{~mm}$ behind the infant cornea, such variations in corneal radius should result in a range of individual variation in $\mathrm{k}$ of about $\pm 12 \%$ around the group-average value. This range of individual variation is in fact about what had been estimated from the actual calibration data collected on a sample of very young infants (Bronson, 1982, Appendix, Figure 4). In summary, by either approach, it seems that when the issue under investigation requires rather precise measurement, separate calibration data should be collected on each individual infant: A $12 \%$ error in estimating $\mathrm{k}$ introduces significant discrepancies when determining the locations of peripheral fixations.

\section{Effects of Changing Comeal Curvature at Different Eccentricities}

As a background for this analysis, consider first a source of nonlinearity in the calibration algorithm that is inherent to the corneal-reflex method. As shown in Figure 5, the displacement of the corneal reflection from the pupil center, $x$, is a function of the sine of the angle $\theta$. The magnitude and direction of this nonlinear "sine effect" when translating raw data (values of $x$ ) into the direction of regard $(\theta)$ can be illustrated by imagining that the parameter linking $\mathrm{x}$ to $\theta$ has been determined from calibration data based on a set of nearcamera fixations and then has been applied via a linear extrapolation to locate fixations directed by the subject to more peripheral parts of the field. It would be found that a fixation directed $10 \mathrm{deg}$ off the camera axis would be calculated to fall at an eccentricity of only $9.9 \mathrm{deg}$. When regard was directed to $20 \mathrm{deg}$ away from the camera, it would be computed to fall at an eccentricity of $19.6 \mathrm{deg}$; and a fixation of $30 \mathrm{deg}$ in the peripheral field would be located at only $28.7 \mathrm{deg}$, an error of some $1.3 \mathrm{deg}$ of visual angle. In brief, it appears that a moderate degree of error might be introduced if one adopts a rather simple linear algorithm for translating raw spot/pupil-center displacements into locus-offixation data. The following analysis, however, indicates that calculations might actually be more accurate if one were to apply the linear-type algorithm.

In the above analysis, the cornea has been considered to be spherical, that is, of constant radius. However, the photokeratoscopic data show that the radius of curvature increases as one moves farther from the optic axis. A consequence of this increasing corneal "flatness" is a relatively more rapid displacement of the reflected spot, for a given change in $\theta$, as the subject looks increas- ingly farther away from the camera location. In effect, in the formula $x=k X \sin \theta$ (see Figure 5), the value of $\mathrm{k}$ increases as $\theta$ gets larger, which will compensate for the decreasing rate of change in the value of $\sin \theta .^{5}$ The net effect can be illustrated by repeating the calculations of the preceding "sine function" analysis, but now with the effects of changing corneal curvature, as well as the sine-function effects, included in the computations. Imagine, as previously, that calibration data have been collected from a set of near-camera fixations, and the raw data from fixations directed to more distant locations then are interpreted under the simplifying assumption that $\theta$ is a linear function of the spot-pupil displacement $x$. The calculations here are based on Mandell's (1967) curvature data, with the cumulative effects of successive 5-deg shifts away from the camera being determined. When the infant is looking $10 \mathrm{deg}$ away from the camera, the calculated location is $10.0 \mathrm{deg}$. When looking at a location $20 \mathrm{deg}$ peripheral to the camera, the computed value is $20.1 \mathrm{deg}$, and for regard directed $30 \mathrm{deg}$ in the periphery, the computed value is 30.2 deg. In brief, for the typical changes in corneal curvature reported by Mandell the sine-function effects and the change in corneal curvature appear to nearly cancel. Therefore, it seems that the more simple linear algorithm for translating spot-pupil discrepancies into direction-of-regard data is to be preferred in infant studies, at least as long as the quality of the calibration data continues to be tenuous.

There is a further consideration: As noted earlier, infant subjects have difficulty in accurately centering a series of fixations on some small stimulus feature. If, however, $\mathrm{x}$ and $\theta$ are related in a nearly linear manner over a wide range around the camera axis, then no complex analytic problems will arise if the limited set of calibration stimuli is spaced rather widely over the visual field. This greater separation will reduce considerably the diluting effects of infant imprecision in the centering of fixations on a small target, thereby producing more accurate calibration data.

\section{Variations in Corneal Curvature at Different Meridians}

If, for a given infant, the radius of corneal curvature should differ significantly between, say, the vertical and horizontal axes of the cornea, then the $\mathrm{k}$ parameter linking $\mathrm{x}$ and $\theta$ would have to be determined separately for the two dimensions. York and Mandell (1968) present photokeratoscopic data bearing on this issue. Of 13 subjects who were under 10 months of age, 5 showed a considerable variation, having differences between the maximum and minimum radii ranging from about .25 to $.50 \mathrm{~mm}$ (among older babies and adults, such relatively large differences in corneal curvature over different meridians proved to be rare). To illustrate the effect of such variation, consider a typical "worst-case" difference of $.35 \mathrm{~mm}$, and a typical mean 
value of $\mathrm{k}$ of $4.6 \mathrm{~mm}$. Then, if calibration data happen to be collected only along the dimension having the larger radius, the estimated eccentricities of fixations directed in the orthogonal direction will be underestimated by about $8 \%$. This results in an error of $.8 \mathrm{deg}$ of visual angle for fixations some $10 \mathrm{deg}$ off the camera axis, and of 2.4-deg errors for fixations directed $30 \mathrm{deg}$ in the periphery. At extreme eccentricities from the camera axis, such "worst-case" errors clearly are not trivial. [Unfortunately, the typical orientations of the maximum or minimum corneal radii in infancy remain to be determined, although in adults it is known that the horizontal meridian of the cornea usually is the flatter (Clark, 1974)].

\section{Summary of Calibration Issues}

From photokeratoscopic data on the shapes of infant cornea, it appears that when some degree of precision is required, (1) calibration data should be collected on each individual infant, (2) the calibration stimuli should be spaced widely over the visual field, with a linear algorithm applied for interpolations to near-camera fixations, and (3) calibration data should be collected over two orthogonal dimensions. Note, however, that these conclusions may be modified if it becomes possible to collect relatively accurate calibration data on infant subjects. Some suggestions for improving procedures here can be found in Bronson (1982). A technique that inight be used to discard the less accurate data from a calibration record has been described by Harris et al. (1981).

\section{REFERENCES}

Abramov, I., Gordon, J., Hendrickson, A., Haintine, L., Dobson, V., \& La Bossiere, E. The retina of the newborn human infant. Science, 1982, 217, 265-267.

Astin, R. N. Development of smooth pursuit in human infants. In D. F. Fisher, R. A. Monty, \& J. W. Senders (Eds.), Eye movements: Cognition and visual perception. Hillsdale, N.J: Erlbaum, 1981.

Bronson, G. W. The scanning patterns of human infants: Implications for visual learning. Norwood, N.J: Ablex, 1982.

Crark, B. A. J. Mean topography of normal corneas. Australian Journal of Optometry, 1974, 57, 107-114.

HaINLINe, L. An automated eye movement recording system for use with human infants. Behavior Research Methods \& Instrumentation, 1981, 13, 20-24. (a)
Hainline, L. Eye movements and form perception in human infants. In D. F. Fisher, R. A. Monty, \& J. W. Senders (Eds.), Eye movements: Cognition and visual perception. Hillsdale, N.J: Erlbaum, 1981. (b)

Harris, C. M., Hainline, L., \& Abramov, I. A method for calibrating an eye-monitoring system for use with infants. Behavior Research Methods \& Instrumentation, 1981, 13, 11-17.

Mandell, R. B. Corneal contour of the human infant. Archives of Ophthalmology, 1967, 77, 345-348.

Sheena, D., \& Borah, J. Compensation for some second order effects to improve eye position measurements. In D. F. Fisher, R. A. Monty, \& J. W. Senders (Eds.), Eye movements: Cognition and visual perception. Hillsdale, N.J: Erlbaum, 1981.

Slater, A. M., \& Findlay, J. M. The measurement of fixation position in the newborn baby. Journal of Experimental Child Psychology, 1972, 14, 349-364.

YoRK, M. A., \& MANDELl, R. B. A new calibration system for photokeratoscopy. Part II: Corneal contour measurements. American Journal of Optometry and Archives of American Academy of Optometry, 1968, 46, 818-825.

Youna, L. R., \& Shenna, D. Survey of eye movement recording methods. Behavior Research Methods \& Instrumentation, 1975, 7, 397-429.

\section{NOTES}

1. This is not exactly true because the fovea is somewhat displaced from the optic axis of the eye. However, since the effect remains constant, it is irrelevant to this and the several following analyses.

2. Note: To avoid distortion, the light rays from a given point on the object should be parallel when passing through the prism. This condition can be met by a proper placement of the unit between camera lenses.

3. The same data indicate that during a saccade the rate of pupil translation will be of about the same magnitude.

4. Indeed, it remains uncertain as to just when the infant fovea has developed to a point at which it becomes appropriate to speak of "foveal fixation" by very young infants; see Abramov, Gordon, Hendrickson, Hainline, Dobson, and La Bossiere (1982).

5. For the cornea to "flatten" smoothly, the center of corneal curvature must move farther back from the cornea, increasing the value of $\mathrm{k}$ by an amount about equal to the increase in radius. (The center of curvature also must shift somewhat off the optic axis, but the effects would be trivial in the present analysis and can be neglected.) The radius of corneal curvature also may increase rather more rapidly on the temporal than on the nasal side of the eye, and the effects appear to differ somewhat among infants (Mandell, 1967, Figure 4). Typical values have been used for the present analysis.

(Received for publication June 22, 1982; revision accepted November 5, 1982.) 